\title{
Identificación inmunohistoquímica de antígenos receptores de angiotensina II en arteria umbilical de alpaca (Vicugna pacos)
}

\author{
Inmunohistochemical identification of angiotensin II antigen receptors in \\ umbilical artery of alpaca (Vicugna pacos)
}

\author{
Emanuel Ayala C. ${ }^{1}$, Alberto Sato S. ${ }^{1,3}$, Nieves Sandoval C. ${ }^{2}$, Miluska Navarrete Z. ${ }^{1}$
}

\section{Resumen}

El objetivo de este trabajo fue identificar los antígenos receptores de angiotensina II en la arteria umbilical de la alpaca (Vicugna pacos). Se trabajó con seis úteros grávidos de alpacas procedentes de comunidades alpaqueras a 4000 msnm en Cusco, Perú, cuyo tiempo de gestación fue estimado por el diámetro biparietal del feto. Se obtuvieron cortes histológicos de $5 \mu \mathrm{m}$ de espesor de cada arteria umbilical. Se aplicó la técnica de inmunohistoquímica (IHQ) Avidina-Biotina-peroxidasa, utilizando reactivos de IHQ y anticuerpos policlonales anti-Angiotensina II. Las láminas fueron analizadas con microscopio binocular Leica DM750 con cámara digital anexada y software LAS 4.7 Leica Macrosystems. La intensidad de marcación se clasificó de manera cualitativa (0: sin marcación; 1 : marcación leve; 2 : marcación moderada; 3 : marcación fuerte). Para el control positivo se empleó muestras de arteria umbilical ovina. Los antígenos receptores de angiotensina II se localizaron en las células musculares lisas de las arterias umbilicales en los tiempos de gestación evaluados. Los antígenos presentaron una intensidad de marcación fuerte, lo que demuestra que la angiotensina ejerce su actividad biológica de vasoconstricción en las células musculares lisas del vaso sanguíneo.

Palabras clave: alpaca; angiotensina; arteria umbilical; inmunohistoquímica

\section{Abstract}

The aim of this study was to identify the angiotensin II receptor antigens in the umbilical artery of the alpaca (Vicugna pacos). Six gravid uteri of alpacas from community farms located at $4000 \mathrm{~m}$ above the sea level in Cusco, Peru were used. The gestation time

\footnotetext{
${ }^{1}$ Laboratorio de Anatomía Animal y Fauna Silvestre, Facultad de Medicina Veterinaria, Universidad Nacional Mayor de San Marcos, Lima, Perú

${ }^{2}$ Laboratorio de Histología, Embriología, y Patología Veterinaria, Facultad de Medicina Veterinaria, Universidad Nacional Mayor de San Marcos, Lima, Perú

${ }^{3}$ E-mail: albertosato2000@yahoo.com
}

Recibido: 13 de junio de 2019

Aceptado para publicación: 20 de septiembre de 2019 
was estimated by the biparietal diameter of the foetus. Histological sections of $5 \mu \mathrm{m}$ thickness were obtained from each umbilical artery. The Avidin-Biotin-peroxidase immunohistochemistry (IHQ) technique was applied, using IHQ reagents and polyclonal anti-Angiotensin II antibodies. The slides were analysed with a Leica DM750 binocular microscope with digital camera attached and LAS 4.7 Leica Macrosystems software. The intensity of marking was classified qualitatively ( 0 : negative; 1 : weak; 2 : moderate; 3 : strong labelling). For the positive control, ovine umbilical artery samples were used. The angiotensin II receptor antigens were in the smooth muscle cells of the umbilical arteries in the various gestation times. The antigens showed a strong labelling intensity, showing that angiotensin exerts its biological activity of vasoconstriction in the smooth muscle cells of the blood vessel.

Key words: alpaca; angiotensin; umbilical artery; inmunohistochemestry

\section{INTRODUCCIÓN}

Las alpacas (Vicugna pacos) habitan mayormente en las tierras altas del Perú, Bolivia, Argentina y Chile, donde las temperaturas caen bajo cero por la noche. Estas especies prefieren baja humedad y altitudes entre 3600 y $4900 \mathrm{~m}$ y convierten con eficiencia los pastos nativos en carne y fibras de alta calidad (Iñiguez y Alem, 1996), convirtiéndolos en el mejor medio de sustento para los habitantes de escasos recursos de los países andinos (Quispe, 2009).

Al habitar en altitudes donde los niveles de oxígeno y la presión atmosférica son reducidos (Iñiguez y Alem, 1996) se estima que la irrigación sanguínea fetal puede tener características histoquímicas propias de la especie. La placenta de la alpaca presenta dos venas, dos arterias y un ducto alantoideo (Barrios et al., 2017). Histológicamente, los camélidos sudamericanos tienen un tipo de placenta denominada epitelio-corial difusa (Dellman, 1993), que descansa en un mesénquima ricamente vascularizado (Olivera et al., 2003) sin placentomas (Iturrizaga et al., 2005).

El desarrollo y crecimiento vascular sanguíneo esta mediado por moléculas de angiotensina (Risau e Ingo, 1995), la cual es responsable de la permeabilidad (Peach,
1981; Woods, 1993), del control de la irrigación (Dvorak et al., 1995: Brameld et al., 2011) y del paso de agua hacia la placenta, regulando de esta manera los fluidos amnióticos y alantoides (Faber y Anderson, 1992). En el humano y en el cerdo, la angiotensina es responsable de la angiogénesis placentaria y del desarrollo fetal durante la gestación (Nielsen et al., 1996).

Estudios relacionados a receptores de angiotensina en los vasos sanguíneos del cordón umbilical de la alpaca son aún motivo de investigación. Por esta razón, el objetivo del presente estudio fue identificar antígenos receptores de angiotensina II en las arterias umbilicales de la alpaca (Vicugna pacos) mediante el uso de la inmunohistoquímica.

\section{Materiales y MéTodos}

\section{Lugar de Ejecución y Animales}

Se emplearon seis úteros grávidos (entre 70 y 270 días de gestación) de alpacas Huacaya, procedentes de comunidades alpaqueras de la zona de Cusco, Perú, ubicadas a $4000 \mathrm{msnm}$, y que formaban parte del estudio «Convenio 097-2014-FONDECYTDE». Los úteros fueron conservados a $8{ }^{\circ} \mathrm{C}$ en una caja refrigerada con hielo y transportados durante $3 \mathrm{~h}$ al Laboratorio de Anato- 
Cuadro 1. Anticuerpos y reactivos empleados para la inmunohistoquímica en arteria umbilical de alpaca

\begin{tabular}{lll}
\hline Anticuerpo & Laboratorio & Dilución \\
\hline $\begin{array}{l}\text { Anticuerpo primario policlonal } \\
\text { Rabbit anti-Human AGTR2. }\end{array}$ & US Biological Life Sciences & $1: 500$ \\
Cat. N ${ }^{\circ}$ P170170 & & \\
$\begin{array}{l}\text { Vectastain ABC KIT. Cat. N. }{ }^{\circ} \\
\text { PK 6100 }\end{array}$ & Vector Laboratories, Inc. & $\begin{array}{l}\text { Listo para uso. Provisto por } \\
\text { el fabricante }\end{array}$ \\
$\begin{array}{l}\text { Reactivo ImmPACT DAB } \\
\text { Peroxidase Substrate }\end{array}$ & Vector Laboratories, Inc. & $\begin{array}{l}30 \mu 1 \text { de cromógeno en 1 ml } \\
\text { de diluyente }\end{array}$ \\
\hline
\end{tabular}

mía Animal y Fauna Silvestre de la Facultad de Medicina Veterinaria (FMV), Universidad Nacional Mayor de San Marcos (UNMSM), en Lima.

\section{Muestras y Mediciones}

Los órganos fueron diseccionados en el laboratorio y los fetos fueron extraídos. El diámetro biparietal fetal (DBP) fue medido con una regla de Vernier para estimar la edad fetal (DBP-0.11376)*47.23287 (Gazitua et al., 2001). Las muestras de arteria se obtuvieron realizando cortes de $2 \mathrm{~cm}$ al inicio del cordón umbilical, próximo al feto. Las muestras fueron conservadas en solución de formalina tamponada al $10 \%$, y procesadas con parafina (bloque de tejido o taco) para el estudio histológico. Cortes de 5 ìm de espesor se hicieron en los bloques y se colocaron en láminas portaobjetos positivados. Los cortes histológicos para la identificación de antígenos de angiotensina y endotelina siguieron el protocolo de inmunohistoquímica $\mathrm{ABC}$ - técnica Avidina - Biotina - Peroxidasa (Doherty et al., 1989; Urpegui et al., 2000), empleando los anticuerpos y reactivos indicados en el Cuadro 1.

\section{Análisis de Láminas}

Las láminas fueron analizadas en el microscopio binocular Leica DM750 con cámara digital ICC50 HD anexada y software de medición LAS 4.7 Leica Macrosystems. Se observó el grado de marcación de los antígenos para angiotensina en arterias, denominándose cualitativamente la intensidad del marcaje como 0: sin marcación, 1: marcación leve $(+), 2$ : marcación moderada $(++)$ y 3: marcación fuerte $(+++)$ (Zanuzzi, 2010).

\section{Términos de Ética}

Los úteros fueron obtenidos de animales descartados por características fenotípicas indeseables para la crianza y que se encontraban destinados al beneficio en el camal local, concordando de esta manera con las normas establecidas por el Comité de Ética y Bienestar Animal de la FMV-UNMSM.

\section{Resultados y Discusión}

\section{Arteria Umbilical}

La arteria umbilical está conformada por tres capas. La primera, denominada túnica- 


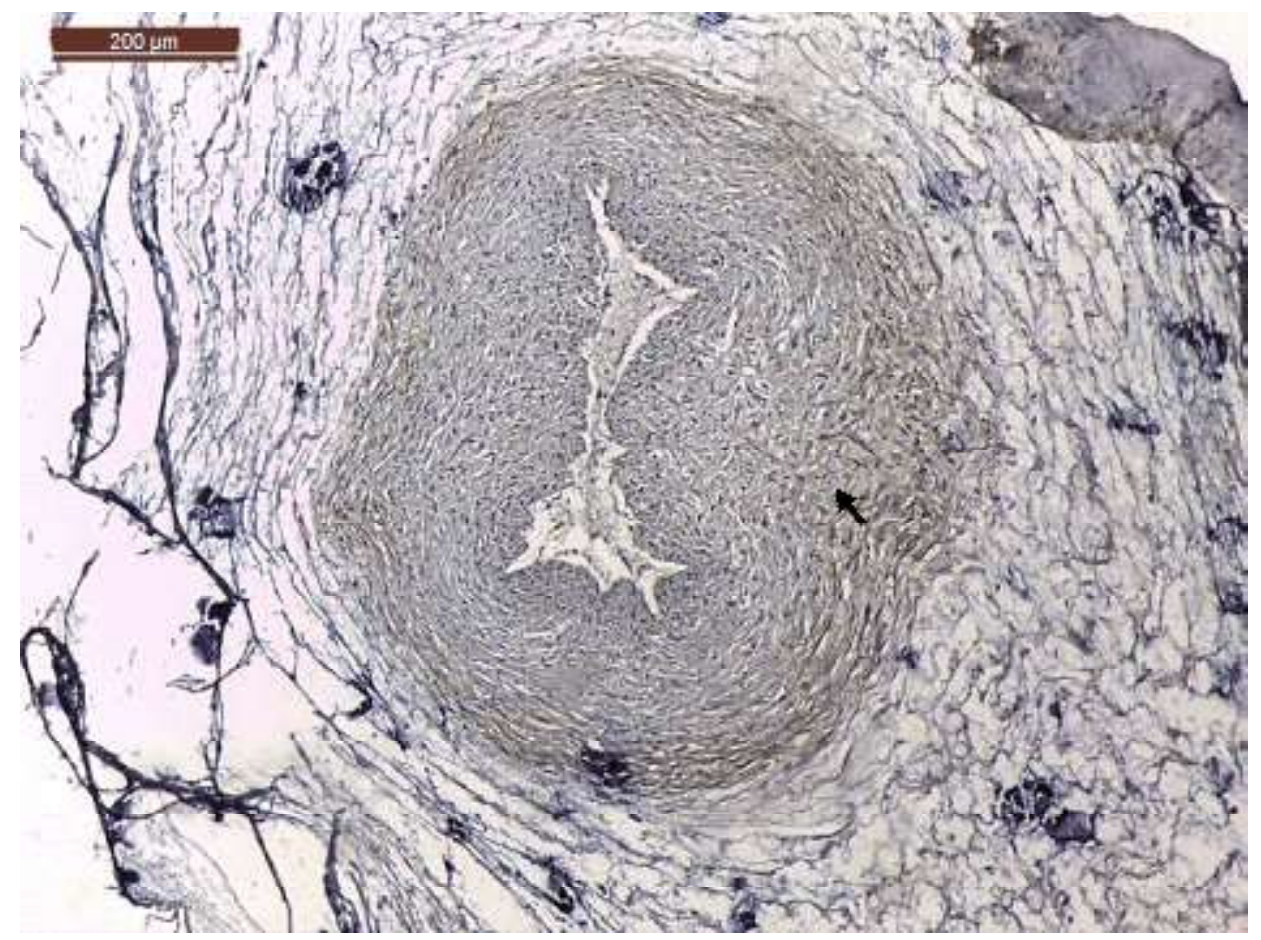

Figura 1. Arteria umbilical de alpaca a los 70 días de gestación. Se señala la intensidad fuerte $(+++)$ del antígeno receptor de angiotensina en las células musculares lisas (IHQ, contraste H\&E 10x)

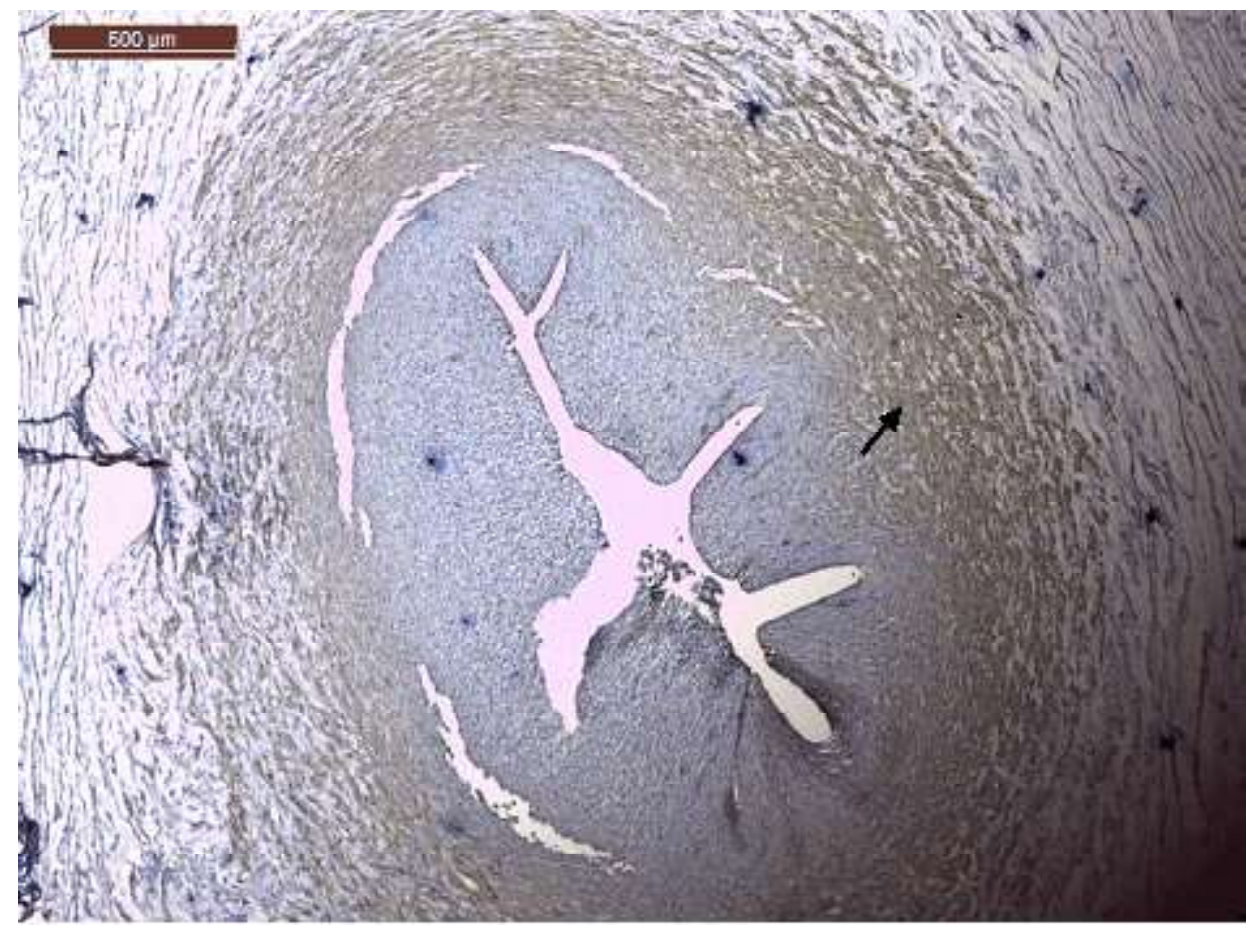

Figura 2. Arteria umbilical de alpaca a los 150 días de gestación. Se señala la intensidad fuerte $(+++)$ de antígenos receptores de angiotensina en las células musculares lisas (IHQ, contraste $\mathrm{H} \& \mathrm{E} 4 \mathrm{x}$ ) 


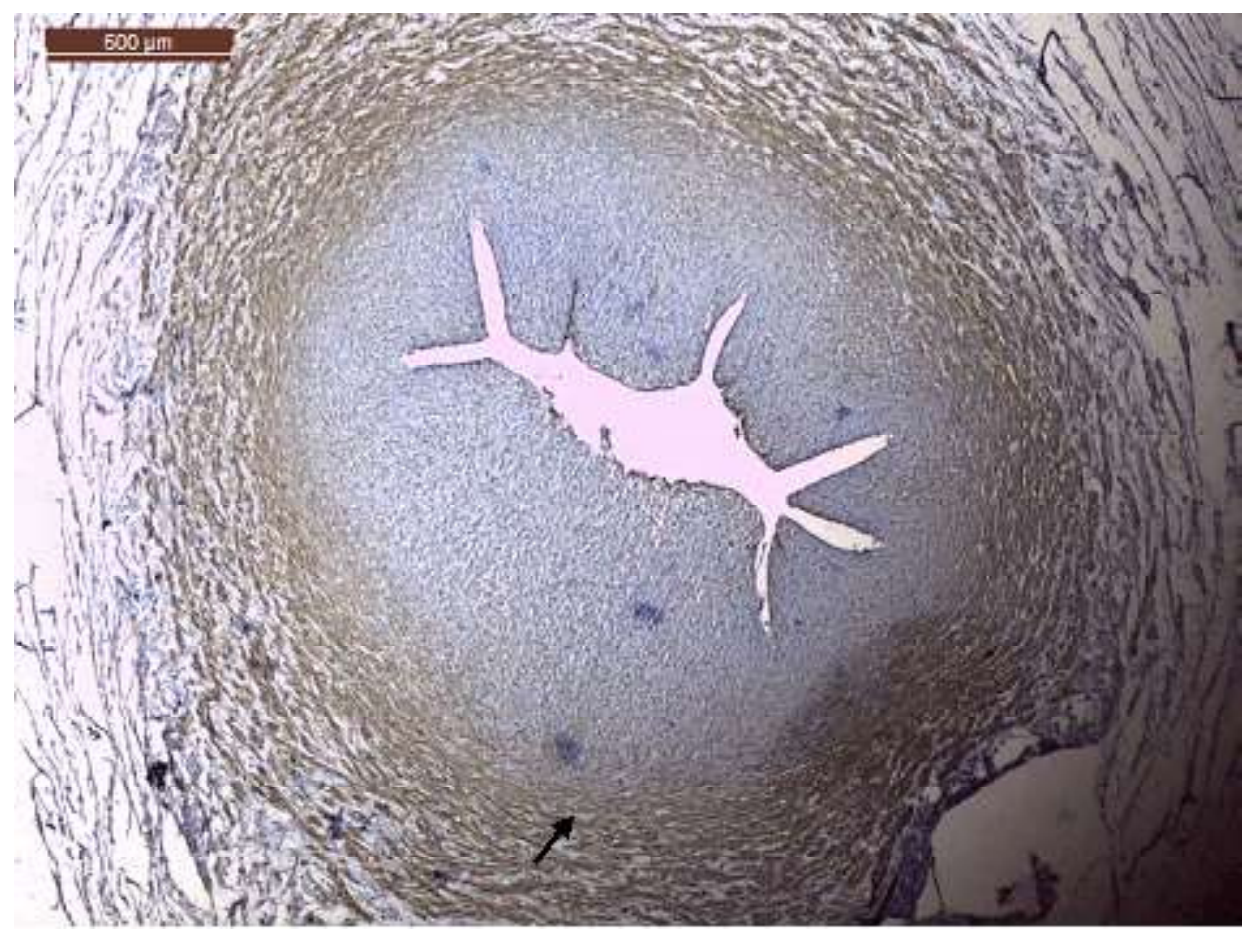

Figura 3. Arteria umbilical de alpaca a los 170 días de gestación. Se señala la intensidad fuerte $(+++)$ de antígenos receptores de angiotensina en las células musculares lisas (IHQ, contraste H\&E 4x)

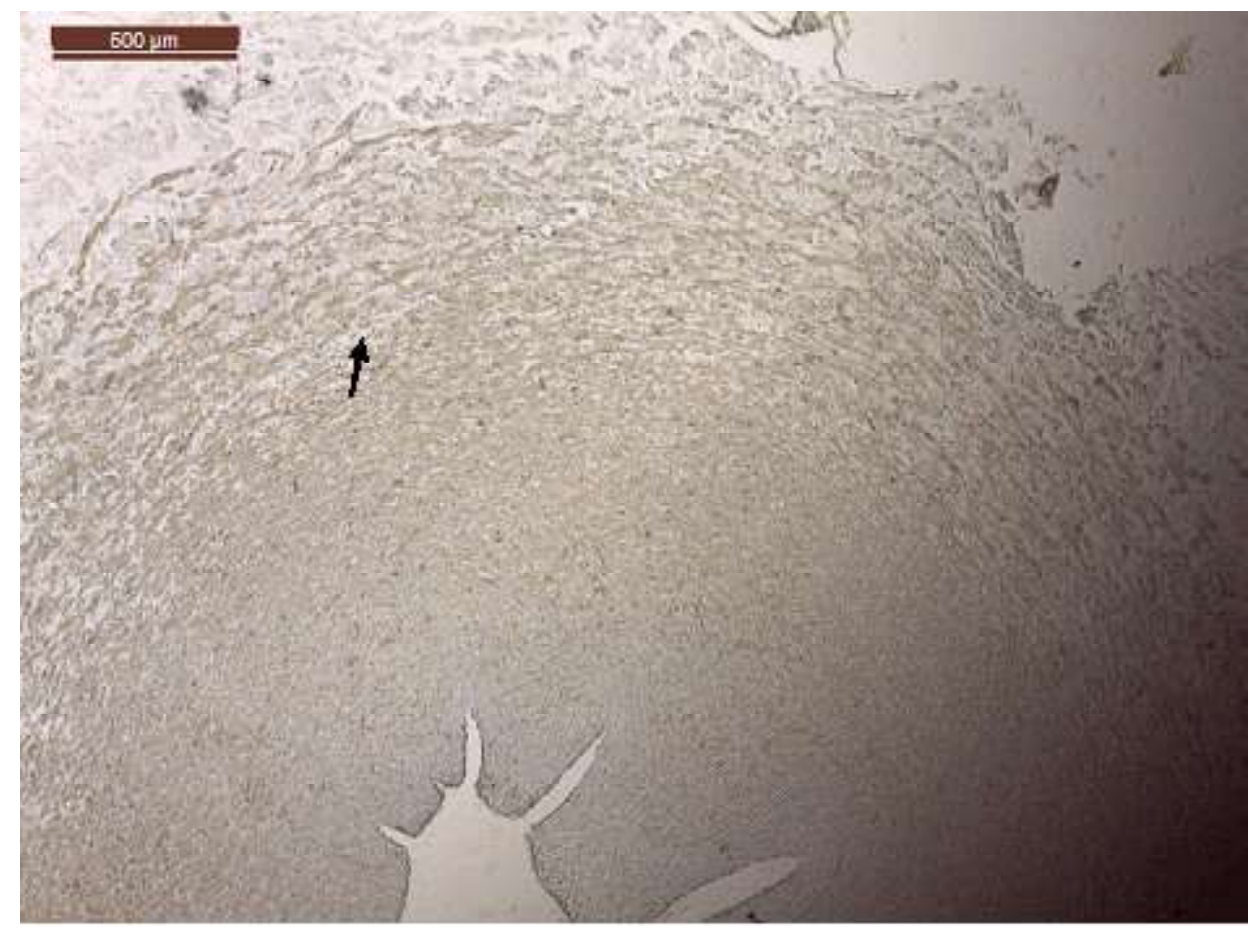

Figura 4. Arteria umbilical de alpaca a los 270 días de gestación. Se señala la intensidad fuerte $(+++)$ de antígenos receptores de angiotensina en las células musculares lisas (IHQ, contraste H\&E 4x) 
interna, compuesta por células endoteliales; la segunda capa se subdivide en una zona compacta y otra esponjosa, donde la zona compacta presenta abundante tejido conectivo entremezclado con escasas células musculares lisas, mientras que la zona esponjosa está conformada por abundantes células musculares lisas que recubren toda la zona compacta. La tercera capa de tejido laxo recubre toda la arteria.

Los resultados de inmunohistoquímica muestran intensidad de marcación fuerte $(+++)$ para antígenos receptores de angiotensina II en la zona esponjosa (Figuras 1-4); es decir, en la zona de mayor presencia de células musculares lisas. La fuerte intensidad de marcación antigénica de angiotensina es atribuida a la presencia de gran cantidad de receptores de angiotensina II en las paredes de las células musculares lisas, los cuales cumplen la función de regular el caudal sanguíneo (Dvorak et al., 1995; Brameld et al., 2011).

La placentación y supervivencia fetal depende de la angiogénesis (Risau e Ingo, 1995), la formación de la red vascular en la placenta (Mackenzie et al., 1999), la circulación fetoplacentaria, y el flujo sanguíneo uteroplacentario está mediado por la angiotensina (Krukier y Pogorelova, 2005). $\mathrm{La}$ angiotensina II es un agente muy potente que incrementa la presión sanguínea al actuar en el músculo liso (Kaschina y Unger, 2003), regula la resistencia vascular y el flujo sanguíneo (Woods, 1993). Aunque, la alpaca habite en grandes altitudes no es ajena a la presencia de receptores de angiotensina y a su actividad biológica que ejerce en las arterias del cordón umbilical. En el presente estudio se ha demostrado que la zona con abundante músculo liso de la arteria umbilical presenta receptores de angiotensina II durante la gestación.

\section{Conclusión}

Los antígenos receptores para angiotensina II se encuentran localizados en las paredes de las células musculares lisas de las arterias umbilicales de alpaca durante la gestación, mostrando a través de la técnica de inmunohistoquímica la presencia de gran cantidad de receptores angiotensina II.

\section{Agradecimientos}

Los autores agradecen al Convenio 0972014-FONDECYT-DE por el financiamiento del presente estudio. Así mismo, al Mg. MVZ Francisco Santos Rueday MV AlexanderChávez Reátegui por el apoyo técnico recibido.

\section{Literatura Citada}

1. Barrios LM, Rodríguez JL, Lopez B. 2017. Histological characterization of umbilical cord in alpaca (Vicugna pacos). Anat Histol Embryol 46: 533539. doi: 10.1111/ahe.12298

2. Brameld JM, Hold R, Broughton Pipkin F. 2011. Regional variation in angiotensin converting enzyme activity in the human placenta. Placenta 32: 906908. doi: 10.1016/j.placenta.2011.07.085

3. Dellman HD. 1993. Textbook of veterinary histology. $4^{\text {th }}$ ed. Philadelphia, USA: Lea \& Febiger. 420 p.

4. Doherty MJ, Russo GG, Jolly HW, Stewart KR. 1989. Immunoenzyme techniques in dermatopathology. J Am Acad Dermatol 20: 827-837. doi: 10.1016/S0190-9622(89)70095-5

5. Dvorak H, Brown L, Detmar M, Dvorak A. 1995. Vascular permeability factor/vascular endothelial growth factor, microvascular hyperpermeability, and angiogenesis. Am J Pathol 146: 1029-1039. 
6. Faber JJ, Anderson DF. 1992. Current topic: water volume of the ovine conceptus. Point of view. Placenta 13: 199-212.

7. Gazitua F, Corradine P, Ferrando G, Raggi L, Parraguez V. 2001. Prediction of gestational age by ultrasonic fetometry in llamas (Lama glama) and alpacas (Lama pacos). Anim Reprod Sci 66: 81-92. doi: 10.1016/01434004(92)90035-R

8. Iñiguez, L, Alem R. 1996. Role of camelids as means of transportation and exchange in the Andean region of Bolivia. World Anim Rev 86: 12-21

9. Iturrizaga D, Verechia F, Santos T, Bombonato P, Teixeira D, Miglino M. 2005. The materno-fetal inter-fetal interface in llama (Lama guanicoe glama). Pesq Vet Bras 27: 221-228.

10. Kaschina E, Unger T. 2003. Angiotensin AT1/AT2 receptors: regulation, signal in and function. Blood Pressure 12: 70-88.

11. Krukier I, Pogorelova T. 2005. Production of vascular endothelial growth factor and endothelin in the placenta and umbilical cord during normal and complicated pregnancy. B Exp Biol MeD 141: 216-218

12. Mackenzie HS, Ziai F, Omer SA, Nadim MK, Taal MW. 1999. Angiotensin receptor blockers in chronic renal disease. The promise of a bright clinical future. $\mathrm{J}$ Am Soc Nephrol 10: 283-286.

13. Nielsen AH, Schauser K, Winther H, Datnzer V, Poulsen K. 1996. Angiotensin II receptors and renin in the porcine uterus: myometrial AT2 and endometrial AT1 receptors are down regulated during gestation. Clin Exp Pharmacol P 24: 309-314. doi: 10.1111/ j.1440-1681.1997.tb01193.x

14. Olivera L, Zago D, Leiser R, Jones C, Bevilacqua E. 2003. Placentation in the alpaca Lama pacos. Anat Embryol 207: 45-67. doi: 10.1007/s00429-0030328-3

15. Peach MJ. 1981. Molecular actions of angiotensin. Biochem Farmachol 30: 2745-2751. doi: 10.1016/0006-2952(81)90410-x

16. Quispe EC. 2009. Producción de fibra de alpaca, llama, vicuña y guanaco en Sudamérica. Anim Genetic Resources Info 45: 1-14. doi: 10.1017/S1014233909990277

17. Risau W, Ingo F. 1995. Vasculogenesis. Annu Rev Cell Dev Bi 11: 73-91 doi: 10.1146/annurev.cb.11.110195.000445

18. Urpegui G, Abenia I, Alfonso C, Sancho S, Escorial S, Vallés V. 2000. Aspectos básicos de la inmunohistoquímica en el carcinoma de laringe. Soc Aragonesa Otorrinolaringol 3: 13-16

19. Woods LL. 1993. Role of angiotensin I1 and prostaglandins in the regulation of uteroplacental blood flow. Am J Physiol 264: 584-590. doi: 10.1152/ ajpregu.1993.264.3.R584

20. Zanuzzi C. 2010. Efectos de la intoxicación con Solanum glaucophyllum (duraznillo blanco) sobre la proliferación, diferenciación y muerte celular en el intestino de conejos. Tesis de Doctorado. Argentina: Univ. Nacional de La Plata. 286 p. 